\title{
LA-UR-20-26495
}

Approved for public release; distribution is unlimited.

Title: Vision-Guided Automation and Assistance

Author(s): $\quad$ Schloen, John Robert

Intended for: $\quad$ ORISE summer student final report

Issued: $\quad$ 2020-08-24 
Disclaimer:

Los Alamos National Laboratory, an affirmative action/equal opportunity employer, is operated by Triad National Security, LLC for the National Nuclear Security Administration of U.S. Department of Energy under contract 89233218CNA000001. By approving this article, the publisher recognizes that the U.S. Government retains nonexclusive, royalty-free license to publish or reproduce the published form of this contribution, or to allow others to do so, for U.S. Government purposes. Los Alamos National Laboratory requests that the publisher identify this article as work performed under the auspices of the U.S. Department of Energy. Los Alamos National Laboratory strongly supports academic freedom and a researcher's right to publish; as an institution, however, the Laboratory does not endorse the viewpoint of a publication or guarantee its technical correctness. 


\section{Vision-Guided Automation and Assistance}

\section{Robert Schloen}

Hosted by LANL Group E-3

Mentor: Dr. Beth Boardman

Mentor's Signature: 


\begin{abstract}
Robotic automation and assistance is an excellent way to increase efficiency and safety when working in a potentially hazardous workspace. However, many traditional automation approaches rely on the precise placement of items to be manipulated. The scope of such automated tasks is also limited to the manipulation of one type of object since there is no way to distinguish between different objects. The addition of a vision pipeline, where robot vision is used to localize and recognize objects, can allow the robot to perform more complex tasks in a variable workspace. In my vision pipeline, a depth camera is used to generate a $3 \mathrm{D}$ point cloud of the workspace. Objects to be manipulated are isolated in the point cloud by filtering out shared surfaces and using Euclidean cluster extraction to group clusters of points. The locations of the objects are then estimated from the point cloud of the isolated objects. The localization of objects with robot vision allows for variable workspaces, reducing the overhead associated with requiring all objects to be precisely placed in the workspace. For object recognition, deep learning is used to train a neural network that classifies objects from the raw point clouds. More complex tasks that require multiple objects to be manipulated differently can be achieved through the vision pipeline. Through its ability to visualize the workspace, robot vision greatly improves efficiency and safety of automated and assistive tasks.
\end{abstract}




\section{Vision-Guided Automation and Assistance}

\section{Introduction}

The goal for this summer's research project was to develop a robot vision pipeline that was able to locate and recognize different objects in a variable workspace. Having the ability to locate and recognize objects would increase the efficiency and safety of tasks in the lab, core tenets of the mission at LANL. Vision would also increase the automated and assistive tasks that could be performed by reducing the overhead needed to set up the task workspace and increasing the number of actions the robot could take when presented with variable stimuli. The objects were located by processing the point cloud generated by the depth camera in the simulated environment. The point cloud was passed through the vision pipeline: a series of filters to isolate the points representing the object so the pose could be estimated from those points. The vision pipeline was then expanded for passing the object's raw point cloud through a trained deep neural network (DNN) to infer and recognize the object. This recognition component was done by training a DNN implementation based on the PointNet DNN on an online dataset and a dataset generated from the simulation. This project was interesting because it used deep learning in order to improve automated tasks, in a way that can also be applied to assistive human-robot interactive tasks.

\section{Description of the Research Project}

The vision pipeline was made up of two main components: localization and recognition. These components were used to divide the project using two milestones. The first milestone was the localization and manipulation of random objects in arbitrary positions in the workspace. The second milestone was the recognition of different objects in the workspace. Once completed, these components were combined to form the full vision pipeline, which helped to increase the efficiency and complexity of automated and assistive tasks. With vision, the robot was no longer limited to tasks that require precise initial placement of objects and could respond appropriately to different types of objects not presented in a set order. This allowed for a more variable and complex workspace.

The remote nature of the internship led to the project being completed entirely in simulation. The first step for milestone one was to set up the simulated environment in which the robot manipulated the objects. The simulation was set up using Gazebo, a physics simulation tool, and RVIZ, a visualization tool. Gazebo, RVIZ, and the robot's control programming were written and integrated using the the Robot Operating System (ROS). The simulation was designed to represent the glovebox environment in which the automated tasks focused on were done. The robotic manipulator was simulated using existing robotic hardware found in the lab, specifically the Motoman SIA5D arm with the Robotiq 85 millimeter gripper attached as the end effector. Cartesian path planning was used when planning trajectories while manipulating objects in order to reduce the risk of collisions. Cartesian path planning created linear trajectories through waypoints, which constrained 
the possible paths the robot could take and limited the risk of collisions. For the objects being manipulated, simple shapes were used as representative stand-ins for the types of objects found in the glovebox. Initially, the shapes included simple cylinders and cubes, with the idea to add more complex shapes as the work continued. Since the project relied on vision, a simple depth camera was simulated. The camera was placed in a fixed position looking down on the workspace so that no objects would be able to occlude any other objects. The fixed top-down view also limited the camera's field of view to the area inside the glovebox, which avoided potential security risks. While the single-camera top-down view was sufficient for the current project, future work might benefit from mounting the main camera, or an added supplementary camera, to the end effector, which would allow the robot a more detailed visualization of specific objects.

With the simulation set up, a point cloud generated from the simulated depth camera was processed. This isolated individual objects and extracted their locations and orientations for manipulation. In order to localize an object, the points describing the object must be isolated. First, a passthrough filter was used to remove points that did not pertain to the workspace for the task. The pass-through filter took a set bounding box, checked the location of all the points in the point cloud relative to the bounding box, and removed points that fell outside the box. In this case, the bounding box surrounded the objects and the surface they were on. The next filter applied was for plane segmentation. Plane segmentation attempted to fit a model of a plane to a small sample of the point cloud, and then checked which points in the entire point cloud fell on the plane. The plane model on which the most points fell could then be used to keep or remove only those points. In our implementation, the plane was removed to further isolate the points corresponding to the objects. The points of the objects were isolated at this point, but in the case that there were multiple objects, the point cloud still could not distinguish between them. Euclidean cluster extraction was used at this point to separate the clusters representing the individual objects. Euclidean cluster extraction essentially found the near neighbors of each point and grouped the points accordingly. With points pertaining to the individual objects isolated, the pose of the objects could be estimated. By comparing the relative position of each point in the object cluster, the pose of the centroid of the object could be estimated. The pose of the object was sent to the robot and the robot was then able to manipulate the object. The first milestone was considered complete when a randomly placed object was successfully located and moved to a new position by the robot. A demonstration of this task is shown in Figure 1.

The next milestone was the recognition of specific objects from a known set of objects. Recognition was achieved through the training of a DNN. The DNN consisted of a series of layers with learnable parameters through which an input was passed resulting in a predicted correct output. When training the DNN the predicted output was compared to the actual correct output, which was provided to the DNN along with the corresponding input. The DNN then randomly iterated through the entire training dataset and optimized the learnable parameters, or weights, based on a loss function that determined how close the DNN's guess was to the actual output. The iteration through the dataset, known as an epoch, was completed a number of times, which allowed the DNN to learn the representations of the input and make better guesses. Each epoch iterated randomly through the dataset so the DNN did not learn relationships based on the order of the inputs. 

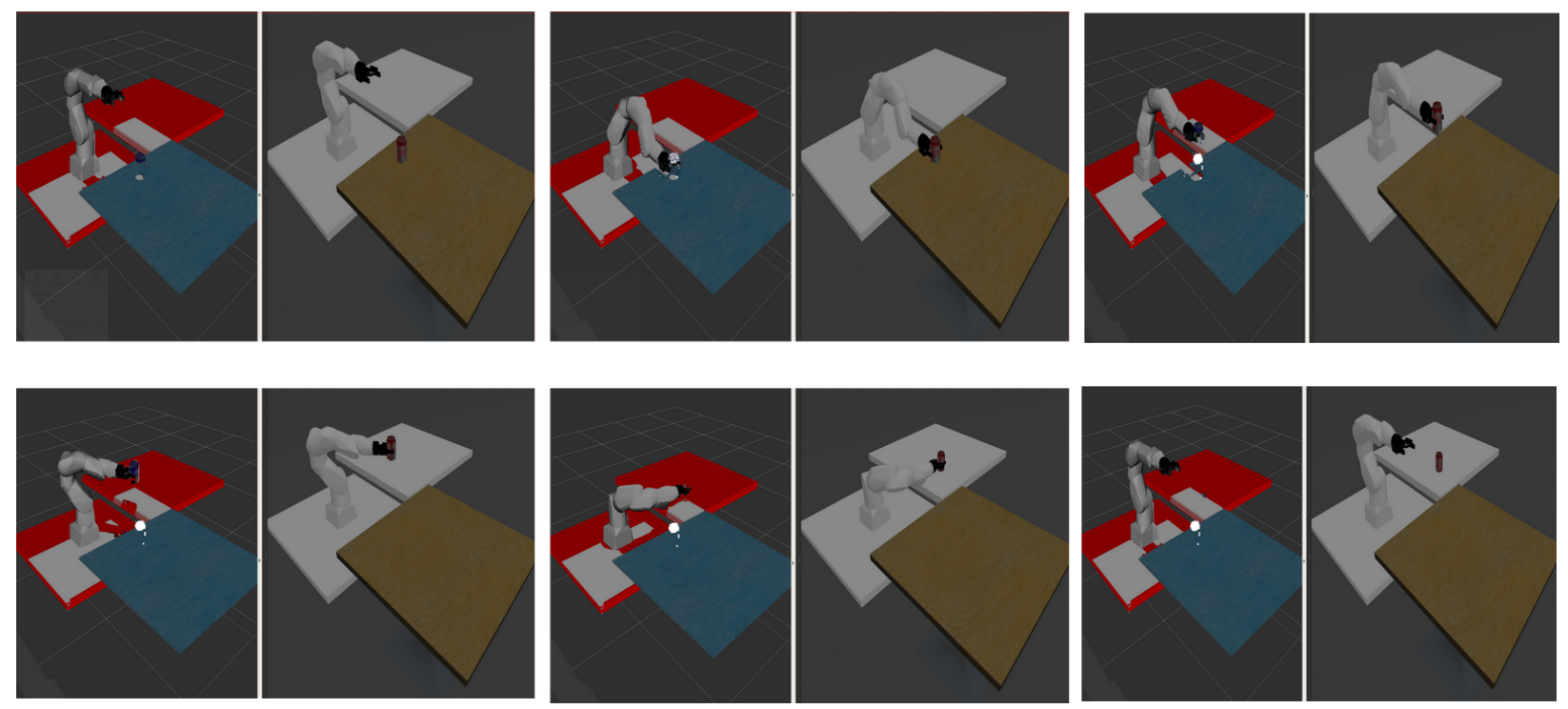

Figure 1: The sequence of images shows a simple pick-and-place task, where the pose of the can was initially unknown. Each panel shows Gazebo on the right and RVIZ on the left.

Once trained, the test dataset, which the DNN had never seen before, was passed through the DNN and the number of correct guesses indicated how well the DNN had been trained. The test dataset did not contribute to the optimization of the learnable parameters and was solely for testing the performance of the already trained model.

Being able to use the raw point cloud was an attractive option for the vision pipeline since it limited the additional computation needed to format the point cloud to pass it through the DNN. However, passing raw point clouds through the DNN presented a few challenges[1]. Point clouds are unstructured since they do not fall on a grid like 2D images. Point clouds are also unordered, in that the points can be listed in any order and return the same result. This is unlike $2 \mathrm{D}$ images where, if the order of the pixels is changed, the image changes. Point clouds can also be sparse and irregularly distributed such that you may have more points in one area of the cloud than another. To help overcome these challenges, we chose to implement a DNN based on the PointNet architecture, which was considered the foundation for DNNs that use raw point clouds[1]. The PointNet architecture consisted mainly of multilayer perceptrons which transformed the feature's dimensionality from 3 ( $\mathrm{x}, \mathrm{y}, \mathrm{z}$ coordinates), to 1024 with shared learnable parameters for each layer. PointNet used max pooling to get the global features since it could be used as a symmetric function, where the order of the inputs does not affect the output. This allowed it to handle the unorderedness of the raw point cloud. In our implementation, as well as that of the PointNet authors', the convolution layers had a kernel size of one and were used to provide the shared learnable parameters for the multilayer perceptrons.

The DNNs required a large amount of data which had enough variation to ensure that the trained 
model was robust to noise and could be generalized to new data it had not seen before. Since it could be challenging to build a dataset from scratch, it could be useful to use an existing dataset that had similar classes of objects. For this reason an existing online dataset, ModelNet[3], was used along with a smaller dataset generated from the simulated environment. The dataset from simulation was created by taking a large number of point clouds with the different objects in various orientations and positions. The reason the larger dataset was helpful, even though it was less specific to the task, was due to the idea of transfer learning. Transfer learning is the idea that the DNN can learn more general relationships from the larger and less specific dataset, which can then be transferred to the smaller dataset. In this way, the general relationships were preserved while the specific relationships were optimized to better fit the target data. The common transfer learning techniques had two main steps. First, the DNN was trained on the large existing dataset. Then, the general relationships that needed to be preserved, the trained weights and parameters of certain layers, were frozen so they would no longer be updated during training. Finally, the DNN was retrained with the smaller dataset. The frozen parameters, which preserved the general relationships from the larger model, remained unchanged after the retraining. As a result, a more generalizable and robust DNN could be trained with the small dataset than if the DNN had been trained solely on the small dataset.

This milestone was to be considered complete once recognition accuracy was suitable and was incorporated into the vision pipeline. While the test accuracy on data not used during training got up to $85 \%$, this proved to be insufficient to produce a useful level of recognition when the trained DNN was incorporated into the vision pipeline. Given more time, further tuning of the hyperparameters would likely increase the DNN's accuracy. Hyperparameters include the optimizer and its parameters, such as learning rate and weight decay, the loss function, and the number of epochs. Additionally, different online datasets could be tested to find one that provides more applicable general relationships, or alternatively, resources permitting, a much larger dataset could be generated from the glovebox setting with the objects of interest. Using a larger dataset from the glovebox setup could reduce the possibility of unhelpful bias introduced by the general dataset and provide better differentiation between similar objects by including more examples. The architecture of the DNN itself could also be modified. While PointNet is a good foundation for raw point clouds, there are likely modifications that could be made to better fit our data. This could include data augmentation techniques that present the point cloud in multiple ways to make the DNN more robust, or changing the types or sizes of layers in the DNN to build more meaningful representations. These are all aspects that could be explored to further improve the DNN's performance.

\section{Contributions Made to the Project}

The project this summer was an individual project, with support and guidance from my mentor. To set up the simulation environment, I added the surfaces, objects, and pre-built depth camera simulation. I combined the existing models of the Motoman SIA5D arm and Robotiq 85mm gripper and configured the Gazebo controllers to behave correctly for this combination. I compiled the necessary packages in ROS to integrate the simulation in Gazebo with the visualization in RVIZ. Using functions from the Point Cloud Library, I developed the initial vision pipeline for localization and manipulation in ROS. I then integrated the pipeline with Gazebo and RVIZ to generate 
and visualize the point clouds and the effect of each step in the clouds in the vision pipeline. Once the localization and manipulation was set up, I implemented a PointNet-based DNN architecture from scratch using the PyTorch C++ API (Libtorch). After I set up the DNN architecture and the dataloader that iterated through the dataset, and then initialized the optimization and loss functions, I trained the DNN. I had time to partially tune some hyperparameters, however more tuning would be needed for better performance. Finally, I added the trained model to the initial vision pipeline and began testing. However, due to the current performance of the trained networks used in the completed pipeline, the pipeline was not reliable and better trained networks were needed. This work was published and presented virtually at the 2020 LANL Student Symposium.

\section{Skills and Knowledge Gained}

Over the course of the project I reinforced some existing skills and learned new ones. In previous coursework and projects, I had used the simulation and visualization tools used for this project, Gazebo and RVIZ, as well as ROS. The use of these tools for this project allowed me to further build upon my previous experience, learn new and better ways of building and integrating models, and learn more about features I had less experience with. The code for this project was mainly written in $\mathrm{C}++$, which was still a relatively new language to me, so the internship was a great opportunity to gain more practical experience with a still somewhat unfamiliar language. The various problems, major and minor, that had come up throughout the project provided opportunities to learn how to approach new problems and solutions in $\mathrm{C}++$. I was also exposed to new topics that I did not have experience with before. Point clouds were a topic I had learned about in coursework but never directly experimented with. Through this project, I learned new tools for capturing and processing point clouds. While I had some previous experience with implementing deep learning solutions, the challenges associated with passing a raw point cloud through a DNN taught me some new ways to handle the data and design the DNN architecture.

\section{Impact on Academic and Career Planning}

The tools and skills I learned and reinforced this summer are valuable for my professional development. My experience has reaffirmed that I want to continue to work on machine learning and artificial intelligence for intelligent assistive or automated robots. My experience this summer has given me a better idea of what a career in research and development might be like. As I finish my Masters program in the fall, my experience this summer will certainly help in determining the next steps in my academic and professional development. 


\section{References}

[1] S. A. Bello, S. Yu, C. Wang, J. M. Adam, and J. Li, "Review: Deep Learning on 3D Point Clouds," Remote Sensing, vol. 12, no. 11, p. 1729, 2020.

[2] R. Q. Charles, H. Su, M. Kaichun, and L. J. Guibas, "PointNet: Deep Learning on Point Sets for 3D Classification and Segmentation," 2017 IEEE Conference on Computer Vision and Pattern Recognition (CVPR), 2017.

[3] Z. Wu, S. Song, A. Khosla, F. Yu, L. Zhang, X. Tang, and J. Xiao, "3D ShapeNets: A deep representation for volumetric shapes," 2015 IEEE Conference on Computer Vision and Pattern Recognition (CVPR), 2015.

[4] A. X. Chang, T. A. Funkhouser, et al. "Shapenet: An information-rich 3d model repository", CoRR abs/1512.03012, 2015. 